\title{
Neuroimmune connections in ovine pharyngeal tonsil: potential site for prion neuroinvasion
}

\author{
Vinciane Toppets • Joelle Piret • Nathalie Kirschvink • \\ Frederic Lantier • Isabelle Lantier • Patricia Berthon • \\ Georges Daube • Laurent Massart • Luc Grobet • \\ Nadine Antoine
}

Received: 29 August 2011 / Accepted: 9 February 2012

(C) Springer-Verlag 2012 light $(70 \mathrm{kDa})$ neurofilaments and of glial fibrillar acidic protein has been semi-quantitatively analysed inside the various compartments of the tonsil. The results show that the most innervated areas are the interfollicular area and the connective tissue located beneath the respiratory epithelium. The existence of rare synapses between follicular dendritic cells and nerve fibres inside the germinal centre indicates that this mechanism of neuroinvasion is possible but, since germinal centres of lymphoid follicles are poorly innervated, other routes of neuroinvasion are likely. The host PRNP genotype does not influence the pattern of innervation in these various tonsil compartments, unlike ageing during which an increase of nerve endings occurs in a zone of high trafficking cells beneath the respiratory epithelium. A minimal age-related increase of innervation inside the lymphoid follicles has also been observed. An increase in nerve fibre density around the lymphoid follicles, in an area rich in mobile cells such as macrophages and dendritic cells capable of capturing and conveying pathogen prion protein (PrPd), might ensure more efficient infectivity, not in the early phase but in the advanced phase of lymphoinvasion after the amplification of PrPd; alternatively, this area might even act as a direct site of entry during neuroinvasion.

Keywords Sheep · Scrapie · Pharyngeal tonsil · Follicular dendritic cell $\cdot$ Innervation

\section{Introduction}

Scrapie, an endemic disease of sheep and goats, belongs to the transmissible spongiform encephalopathies (TSEs), a family of neurodegenerative diseases affecting humans and animals. The TSEs also include Creutzfeld-Jakob disease (CJD) and its 
variant (vCJD) in humans, bovine spongiform encephalopathy (BSE) in cattle and chronic wasting disease (CWD) in mule deer. They are caused by the aggregation of aberrantly folded "prion protein", initially described by Griffith (1967) and refined by Prusiner (1982). This pathogen protein, PrPd, is an isoform of a host-encoded protein, PrPc, physiologically expressed by most cells such as nerve, lymphoid and muscular cells (Horiuchi et al. 1995). Expression of this normal cell protein is essential to TSE pathogenesis. In contact with PrPd, PrPc undergoes a conformational change leading to pathogenic conversion (Weissmann 2004).

In sheep, the genotype of the host modulates susceptibility/resistance to scrapie, which is dependent on various polymorphisms throughout the PrP gene (PRNP). Mutations at positions 136, 154 and 171 of the $\operatorname{PrP}$ chain strongly influence the susceptibility to scrapie. Among the possible genotypes derivable from this combination, the ARR/ARR genotype is strongly associated with resistance to scrapie, whereas VRQ/VRQ is clearly associated with susceptibility (Baylis et al. 2004). Depending on the breed of the sheep, the ARQ/ARQ genotype can be associated with resistance or with susceptibility, as in Suffolk (Hunter et al. 1997) and Texel (Baylis et al. 2002) breeds.

Dissemination of prions into the environment can occur from several biological materials, e.g. infectious placentas (Race et al. 1998), saliva (Vascellari et al. 2007), urine (Seeger et al. 2005) and excreta (Miller et al. 2004). Although the alimentary tract has been suggested as the most likely port of entry in natural scrapie, several other experimentally potent routes of infectivity have been identified (Bessen et al. 2009). In animals, the olfactory system is important in social behaviour, in the search for food and in the exploration of the environment. Recent studies have focused on the role of the nasal cavity not only as a source of infection through the excretion of PrPd in nasal secretions (Bessen et al. 2010) but also as an efficient route for prion uptake and systemic dissemination. Intranasal inoculation of a scrapie strain in hamsters leads to the deposition of the pathological prion protein in the nasal associated lymphoid tissue (NALT) several weeks before detection in the vagus or trigeminal nerve nucleus in the central nervous system (CNS; Sbriccoli et al. 2009). The mechanism of neuroinvasion by prions from the NALT remains unclear. Two pathways are suspected: (1) lymphatic or haematogenous routes (Hunter et al. 2002; Halliday et al. 2005) and (2) "retrograde neuroinvasion" sustained by contact that is initiated during the preclinical phase and that occurs between PrPdreplicating cells in the lymphoreticular system (LRS) and nerve endings, followed by retrograde transport along efferent nerve fibres allowing propagation to the CNS (Van Keulen et al. 2002). In this second mechanism, the innervation of the NALT appears to be a rate-limiting step in the pathway of neuroinvasion after intranasal infection.
The pharyngeal tonsil, located in the caudal part of the pharyngeal septum, contains the largest volume of NALT and the largest epithelial surface of the nasopharyngeal parts of Waldeyer's ring (Casteleyn et al. 2007). Ultramicroscopic examination of the epithelium of the pharyngeal tonsil has revealed the presence of $\mathrm{M}$ cells implicated in the uptake and sampling of foreign antigens and microorganisms (Casteleyn et al. 2010; Kraehenbuhl and Neutra 2000). According to these anatomical and histological characteristics, one is tempted to speculate that the pharyngeal tonsil is an important reservoir for prion pathogen.

In this work, we have evaluated semi-quantitatively the topography of the peripheral nervous system in the lymphoid and non-lymphoid compartments of pharyngeal tonsils. For this analysis, the following variables have been taken into account: the genotype and the age of the sheep. We have highlighted neuroimmune connections between follicular dendritic cells (FDCs), strongly implicated in PrPd amplification (Jeffrey et al. 2000; Mabbott et al. 2000) and nerve fibres in the lymph follicles of pharyngeal tonsils. This observation supports their possible involvement in the neuroinvasion process and sustains the hypothesis of "retrograde neuroinvasion" from the NALT.

\section{Materials and methods}

Animals

Scrapie-free sheep $(n=17)$ were classified into four groups according to their genotype and age: (1) French Texel lambs, 4-6 months old, of the ARR/ARR genotype $(n=6)$, (2) French Texel lambs, 4-6 months old, of the ARR/VRQ genotype ( $n=4)$; (3) old sheep, 24-30 months old, comprising a resistant French Texel of the ARR/ARH genotype $(n=1)$ and resistant Suffolks of the ARR/ARR genotype $(n=3)$; (4) old susceptible French Texels of the ARQ/ARQ genotype $(n=1)$ and ARR/VRQ genotype $(n=1)$ and a Suffolk of the ARQ/ARQ genotype $(n=1)$.

Sample collection

Ovines were killed at a local abattoir and pharyngeal tonsils were removed from the nasopharynx. Sections of the medial part of each tonsil were immersed in Tissue-Tek OCT embedding medium (SAKURA, Zouterwoude, The Netherlands), snap-frozen and stored at $-20^{\circ} \mathrm{C}$.

\section{Cryosections}

Serial transverse sections $(10 \mu \mathrm{m}$ thick) of the medial part of the tonsil were cut at $-15^{\circ} \mathrm{C}$ with a microtome (MICRON HM $500 \mathrm{OM}$ ) and mounted on glass slides coated with poly- 
L-lysine (Sigma, St Louis, Mo., USA), air-dried, fixed in acetone for $10 \mathrm{~min}$ at $4{ }^{\circ} \mathrm{C}$ and then stored at $-80^{\circ} \mathrm{C}$ until use.

\section{Antibodies}

The FDCs were stained with a mouse anti-bovine monoclonal antibody (undiluted hybridoma supernatant), FDC-B1, kindly provided by F. Melot (Melot et al. 2004).

Nerve fibres were labelled with antibodies directed against:

- Intermediate neurofilaments heavy (NFH): rabbit antibovine NFH 200 kDa (1/1000; AbD Serotec, Dusseldorf, Germany);

- Intermediate neurofilaments light (NFL): rabbit antihuman NFL $70 \mathrm{kDa}(1 / 1000$; AbD Serotec);

- Schwann cells: rabbit anti-cow glial fibrillar acidic protein (GFAP; 1/1000; Dako, Glostrup, Denmark).

$\mathrm{NF}$ or neuron-specific intermediate filaments are major cytoskeletal elements in neurons (Gotow 2000). They are the most abundant structural components in myelinated axons, whereas they are sparse in the perikarya and dendrites (Lee and Cleveland 1996). NF in axons are essential for conduction velocity.

GFAP is a monomeric intermediate filament protein expressed in the cytoskeleton of mature astrocytes and outside the CNS in mature non-myelin-forming Schwann cells (Notturno et al. 2008). These proteins are not found, or only at a much lower level, in myelin-forming Schwann cells (Jessen et al. 1990). In order to test the specificity of the monoclonal antibodies used, the samples were incubated with irrelevant antibodies. Negative controls were obtained by incubating samples with secondary antibodies only.

\section{Immunoperoxidase staining}

Cryosections were rehydrated and incubated for $1 \mathrm{~h}$ at room temperature with primary antibodies. A secondary conjugated species-specific immunoglobulin peroxidase-labelled polymer (Amplification EnVision System-horseradish peroxidase [HRP]; Dako, Glostrup, Denmark) was applied on cryosections for $30 \mathrm{~min}$ at room temperature. Peroxidase activity was revealed with 9-ethyl-3-aminocarbazole (Zymed, San Francisco, Calif., USA) combined with $\mathrm{H}_{2} \mathrm{O}_{2}$ as substrate.

\section{Immunofluorescent staining}

Double-immunofluorescent labelling was performed to detect simultaneously FDCs and nerve fibres. Each incubation period with the selected antibodies was performed at room temperature for $1 \mathrm{~h}$. Incubations with secondary antibodies were carried out in the dark. After rehydration, sections were first incubated with FDC-B1 and then rinsed in phosphate- buffered saline. Primary antibodies were revealed with a goat anti-mouse antibody conjugated to Alexa 594 (Molecular Probes, Leiden, The Netherlands) and diluted at 1/4000. Anti-GFAP or anti-NFH antibodies were then applied and revealed with an Alexa-488-conjugated goat anti-rabbit antibody (Molecular Probes) diluted at 1/4000 and 1/8000, respectively.

\section{Confocal analysis}

All samples were observed with a Leica (Germany) SP2 confocal microscope. Cryosections of $10 \mu \mathrm{m}$ were scanned at their best fluorescent zone, which was divided into 15 sections. Each section was analysed. Virtual colours were attributed for detection channels: red for FDCs and green for GFAP-positive $(\mathrm{GFAP}+)$ or NFH+ nerve fibres. The overlap of these virtual colours appeared in yellow and drew our attention to possible contact between the labelled structures. Spectral analysis of the colour channel allowed us to confirm areas of contact.

\section{Semi-quantitative morphometrical analysis}

A semi-quantitative analysis of the distribution of the immunoperoxidase-labelled nerve fibres was assessed throughout the tissue section in the following pharyngeal tonsil compartments: surface epithelium, lamina propria, interfollicular area, mantle zone (MZ) and germinal centre (GC) of the follicles and loose connective tissue constituting the central axis of the tonsil. The presence of labelled nerve fibres in each zone was scored from 0 to 5 on the basis of the following criteria: $0=$ absence of labelled fibre (LF); $<1=$ less than $1 \mathrm{LF} ; 1=$ from 1 to $5 \mathrm{LF} ; 2=$ from 6 to $15 \mathrm{LF} ; 3=$ from 16 to $30 \mathrm{LF} ; 4=$ from 31 to $50 \mathrm{LF} ; 5=$ more than $51 \mathrm{LF}$. Numbers of follicles within each section were counted. In order to standardise our scoring method, we analysed the various compartments in our sections in total and introduced a correction in the statistical analyses. For each section, a statistical covariable was created that took into account the surface and perimeter of (1) the lymphoid compartments and (2) the connective tissue filling the central axis, respectively and (3) the number of follicles in each tissue section. The correction of the data by using such co-variables avoided the variations in the counting linked, for example, to the higher surface area of lymphoid tissue. Six slides per tonsil were randomly selected among the serial transversal sections cut in the middle part of each tonsil. The tissue sections were immunolabelled and analysed blind to the age and the genotype of the sheep.

Statistical analyses

Statistical tests were performed by using the Statistical Analysis System (SAS 9.1.3, service pack 4). The data were 
analysed by a logistic procedure measuring the influence of the independent variables (age and genotype) on dependent variables (GFAP,$+ \mathrm{NFH}+$ and NFL + nerves fibres). The effect was significant if the probability was lower than 0.05 .

\section{Results}

Postulating that (1) the upper respiratory tract and most likely the pharyngeal tonsil serve as a natural site of entry for scrapie agent, (2) FDCs, resident cells of the GC, are implicated in the retention and replication of scrapie agent and (3) nerve fibres probably act as the initial site of neuroinvasion for prions, we examined the innervation pattern of the ovine pharyngeal tonsil and focused our attention on the neuro-FDC interface.

Topography and semi-quantitative analysis of nerve fibres in pharyngeal tonsil

The ovine pharyngeal tonsil located in the caudal part of the pharyngeal septum was observed to be unique, had an approximate size of $3 \mathrm{~cm}$ in length and $1.5 \mathrm{~cm}$ in width and was lined on its external surface by many longitudinal exophytic folds.

Pharyngeal tonsils could be histologically separated into two areas: lymphoid and non-lymphoid compartments (Fig. 1). The non-lymphoid compartments consisted into a central axis, which was filled by loose connective tissue rich in adipose cells and that contained numerous blood and lymphatic vessels; and the deeply folded pseudostratified

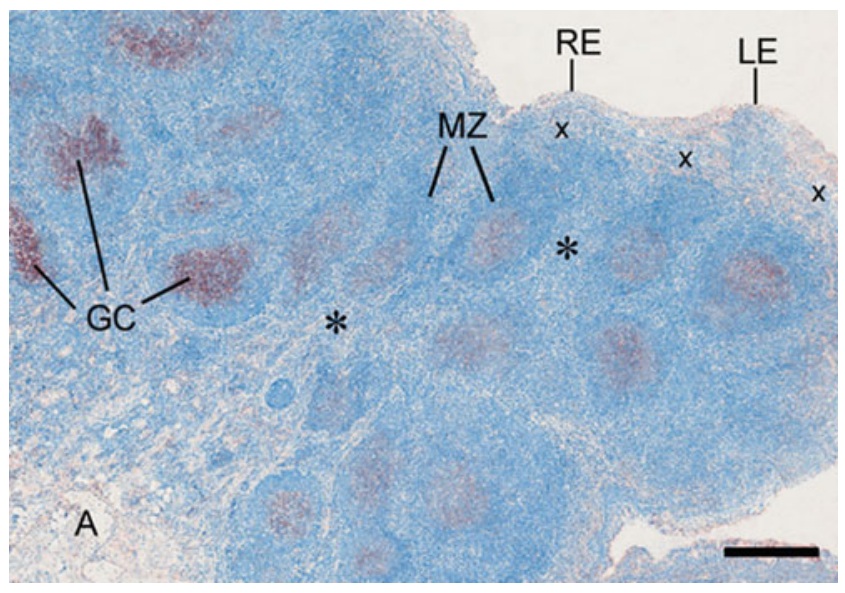

Fig. 1 Lymphoid and non-lymphoid compartments of the ovine pharyngeal tonsil. Cryosection immunolabelled with monoclonal antibody (moAb) FDC-B1. Counterstaining with haematoxylin. The pattern of innervation inside the tonsils was evaluated by semi-quantitative scoring in the various compartments ( $A$ central axis of the tonsil, asterisks interfollicular area, $G C$ germinal centre of the lymphoid follicle containing the follicular dendritic cells labelled in red, $M Z$ mantle zone, $R E$ respiratory epithelium, Le lymphoepithelial tissue, $X$ sub-epithelial connective tissue). Bar $500 \mu \mathrm{m}$ ciliary epithelium covering the luminal surface of the tonsil. The lymphoid compartments consisted into the lymphoid follicles composed of a GC, with resident FDCs in the light zone, clearly identified in Fig. 1 by immunostaining with FDC-B1 antibody and an MZ of naive B cells facing the epithelium; the interfollicular area lying around the lymphoid follicles; and the connective tissue, which lay under the respiratory epithelium and that was highly infiltrated by immune cells. In some locations, the epithelium was invaded by lymphoid cells, thereby losing its typical structure and providing a route to the reticular lymphoepithelium.

The mean scoring of the innervation in these various compartments of the pharyngeal tonsil is presented in Table 1.

In the central axis of the tonsil, many thick bundles of $\mathrm{GFAP}+$ and $\mathrm{NFH}+$ nerve fibres and isolated labelled fibres

Table 1 Mean scoring of innervation in the various compartments of the pharyngeal tonsil in sheep of known age and genotype (RES resistant, SUSC susceptible). The nervous fibres were abundant in the loose collagenous tissue constituting the central axis of the tonsil and in the interfollicular area. Conversely, scoring of innervation was low in the germinal centre $(G C)$ and mantle zone $(M Z)$ of the lymphoid follicles and absent in the respiratory epithelium. If we compare the scoring of innervation in the connective tissue $(C T)$ underlying the respiratory epithelium, we see that the scoring was higher in sheep older than 36 months. The statistical analysis applied to the results reveals that the increase of innervation is only influenced by age, regardless of genotype (scoring: 0 absence of labelled fibre (LF), $<1$ less than $1 \mathrm{LF}, 11-5 \mathrm{LF}, 26-15 \mathrm{LF}, 316-30 \mathrm{LF}, 431-50 \mathrm{LF}, 5$ more than $51 \mathrm{LF}$ )

\begin{tabular}{|c|c|c|c|c|c|}
\hline \multirow[t]{3}{*}{ Nerve markers } & \multirow[t]{3}{*}{ Tonsil compartments } & \multicolumn{4}{|c|}{ Age and genotype } \\
\hline & & \multicolumn{2}{|c|}{$\leq 6$ months } & \multicolumn{2}{|c|}{$\geq 36$ months } \\
\hline & & RES & SUSC & RES & SUSC \\
\hline \multirow[t]{6}{*}{ GFAP } & $\mathrm{GC}$ & $<1$ & 1 & 1 & 1 \\
\hline & $\mathrm{MZ}$ & 1 & 1 & 1 & 1 \\
\hline & Central axis & 4 & 5 & 5 & 5 \\
\hline & Interfollicular area & 4 & 5 & 5 & 5 \\
\hline & Sub-epithelial CT & 1 & 1 & 4 & 3 \\
\hline & Respiratory epithelium & $<1$ & 0 & $<1$ & $<1$ \\
\hline \multirow[t]{6}{*}{ NFH } & GC & $<1$ & 1 & 1 & 2 \\
\hline & $\mathrm{MZ}$ & $<1$ & $<1$ & 1 & 1 \\
\hline & Central axis & 4 & 5 & 5 & 5 \\
\hline & Interfollicular area & 3 & 4 & 4 & 4 \\
\hline & Sub-epithelial CT & 1 & 1 & 3 & 2 \\
\hline & Respiratory epithelium & $<1$ & $<1$ & 1 & $<1$ \\
\hline \multirow[t]{6}{*}{ NFL } & $\mathrm{GC}$ & $<1$ & $<1$ & $<1$ & $<1$ \\
\hline & $\mathrm{MZ}$ & $<1$ & $<1$ & $<1$ & $<1$ \\
\hline & Central axis & 3 & 3 & 3 & 4 \\
\hline & Interfollicular area & 2 & 1 & 2 & 1 \\
\hline & Sub-epithelial CT & 1 & $<1$ & 2 & 1 \\
\hline & Respiratory epithelium & $<1$ & 0 & 1 & 0 \\
\hline
\end{tabular}


were present (score: 4-5). Some of them were seen along and around the muscular wall of the blood vessels. The GFAP+ and NFH+ fibres were located in all the lymphoid compartments in variable proportions but only as sections of isolated fibres. They were abundant in the interfollicular area (score: 35 ), especially under and around the lymphoid follicles, their number tending to decrease towards the lymphoid tissues underlying the pseudostratified ciliary epithelium (Fig. 2a, b). The presence of nerve fibres inside the epithelium was rare (score: $0-1$ ). Some sectioned NFH+ fibres were unusually observed inside the lymphoepithelial tissue.

The topographic partitioning of the NFL+ fibres was the same as that for the GFAP+ and NFH+ fibres; however, their number was definitely lower in each analysed compartment.

Analysis of the innervation pattern highlighted that the lymphoid follicles were poorly innervated by GFAP+, NFH + (score: $<1-2$ ) and NFL+ fibres (score: $<1)$. When they were present, small sections of isolated fibres were detected in the GC and/or in the MZ of the follicles (Fig. 2c, d).

Taking into account all the pharyngeal tonsils analysed, the mean percentage of innervated GC was $3.15 \%$ for GFAP + fibres, $5.36 \%$ for NFH+ fibres and $0.80 \%$ for NFL fibres. However, in one sheep, which was younger than 6 months, the percentage of follicles innervated by NFH+ fibres was $25 \%$ higher than the mean (data not shown).

Regarding the innervation of the MZ without the presence of fibres in the GC, only $5.04 \%$ of the follicles contained GFAP+ fibres in this compartment, $3.47 \%$ contained NFH+ fibres and $0.56 \%$ contained NFL+ fibres.
Effect of age and genotype on innervation rate

Logistic regression tests were carried out on the results to determine the potential effect of age and genotype on the collected scoring of innervation and on the percentage of innervated GC and MZ.

\section{Effect of age}

The statistical analysis applied to the results of the semiquantitative scoring of innervation in each compartment revealed that the number of GFAP + and $\mathrm{NFH}+$ fibres was only influenced by age with regard to the connective tissue underlying the respiratory epithelium. The scoring of innervation was significantly lower in the pharyngeal tonsils of sheep younger than 6 months when compared with sheep older than 30 months, regardless of genotype.

Taking into account the age of sheep within the groups of genotype resistant or susceptible towards scrapie, we observed that the percentages of GC and MZ innervated were lower in animals younger than 6 months when compared with sheep older than 36 months (Figs. 3, 4).

\section{Effect of genotype}

Considering the genotype only, even if the groups of sheep with the susceptible genotype seemed to have a higher percentage of innervation in the GC and MZ, the
Fig. 2 Immunolabelling of ovine pharyngeal tonsillar cryosections by polyclonal antibodies (poAb): antineurofilament $\mathrm{H}(\mathbf{a}, \mathbf{c})$ and antiglial fibrillar acidic protein (b, d). Counterstaining with haematoxylin. a Abundant GFAP+ and $\mathrm{NFH}+$ fibres were present in the loose collagenous connective tissue filling the central axis of the pharyngeal tonsil. Some of them followed the blood vessels. Isolated fibres ran through the interfollicular area, especially under and between the lymphoid follicles. Bar $100 \mu \mathrm{m}$. b GFAP+ fibres in the lymphoid tissues underlying the pseudostratified ciliary epithelium. Bar $100 \mu \mathrm{m}$. c, $\mathbf{d} \mathrm{NFH}+$ fibres (c) and GFAP+ fibres (d) labelled in the GC of lymphoid follicles. In this compartment, the fibre sections were rare, short and isolated. Insets Higher magnification view of indicated region. Bar $200 \mu \mathrm{m}$

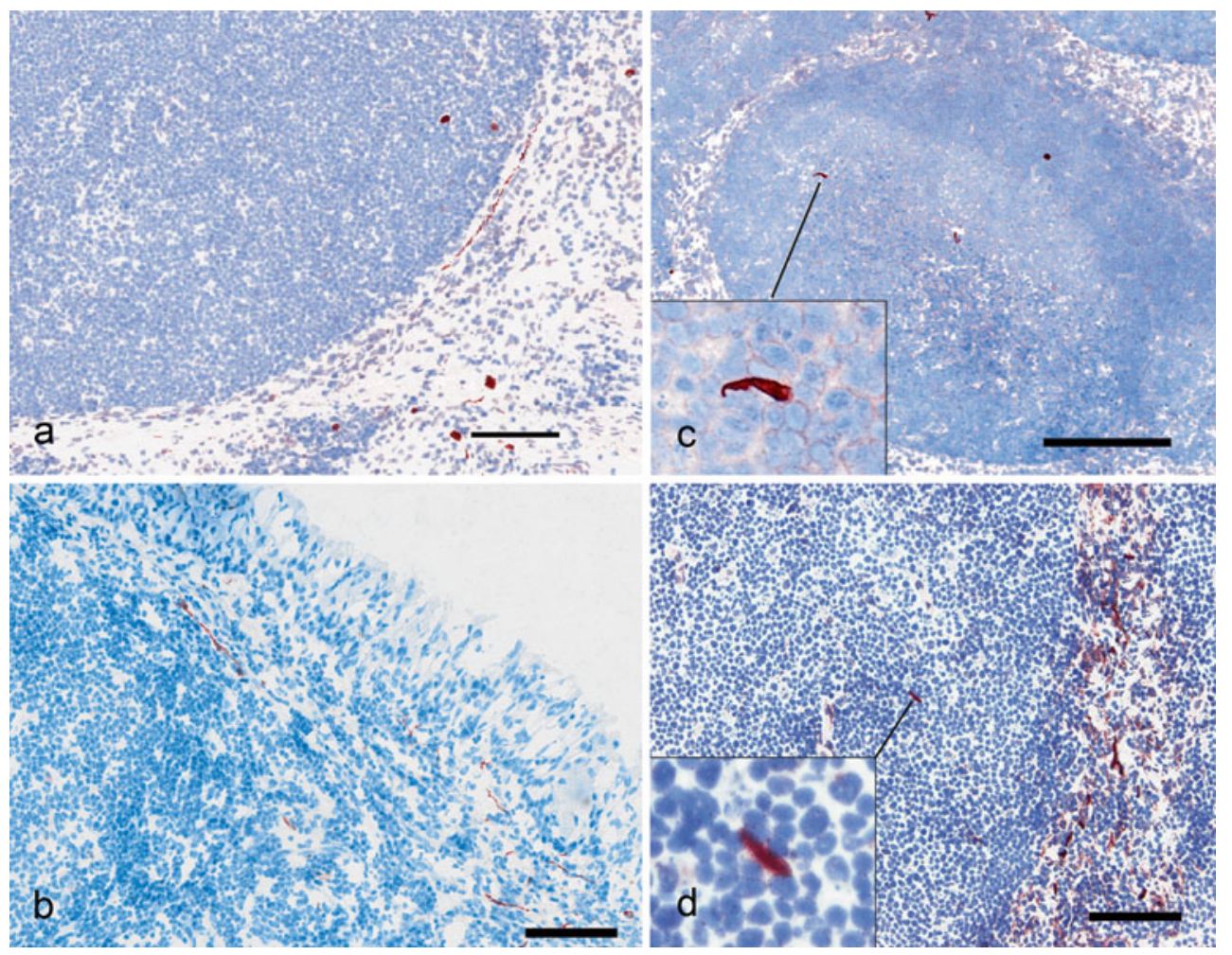




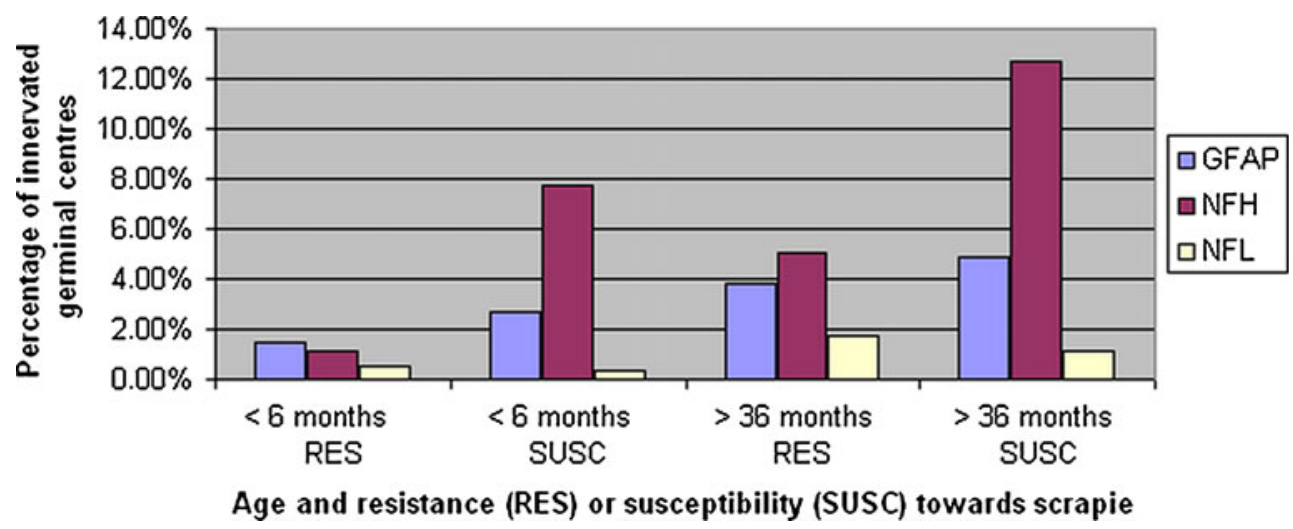

Fig. 3 Percentage of GC innervated by GFAP + , NFH+ or NFL+ fibres. Analysis of the innervation pattern highlighted that the lymphoid follicles were poorly innervated by GFAP + , NFH+ and NFL + fibres (respectively, $3.15 \%, 5.36 \%$ and $0.80 \%$ ). Whatever the genotype, the percentage of GC innervated was lower in animals younger

logistic regression demonstrated that this difference was not significant.

\section{Neuroimmune interface}

The topographic analysis of the innervation pattern revealed the presence of NFH+ and GFAP + nerve fibres in the GC of the lymphoid follicles in which the FDCs were located. Therefore, we analysed, by confocal microscopy, doublefluorescent immunolabelling of FDCs and NFH+ or GFAP+ nerve fibres. The analysis of the spectra (red for FDCs and green for $\mathrm{NFH}+$ or GFAP+ fibres) at the precise point of superimposition of these virtual colours (yellow point) revealed a perfect alignment of the peaks of intensity for each colour, thus establishing the ultra-close co-localisation between the cytoplasmic extensions of the FDCs and GFAP+ (Fig. 5) or NFH+ (Fig. 6) fibres present in the GC and/or in the MZ of some follicles. We could conclude that, even if the innervation rate inside the GC (respectively, 3.15\% for GFAP ,$+ 5.36 \%$ for $\mathrm{NFH}+$ and $0.80 \% \mathrm{NFL}+$ nerve fibres) and the $\mathrm{MZ}$ (respectively, 5.04\% for GFAP+, 3.47\% for $\mathrm{NFH}+$ and $0.56 \%$ NFL + nerve fibres) was low, neuroimmune junctions between FDCs and NFH+ or GFAP+ fibres were detectable. than 6 months when compared with sheep older than 30 months. This difference, although small, was significant. Even if the groups of sheep with the susceptible genotype seemed to have a higher percentage of innervated GC than the groups of resistant genotype, the statistical analyses indicated that this difference was not significant

\section{Discussion}

Innervation pattern in pharyngeal tonsil: potential mechanism of neuroinvasion for PrPd

In natural scrapie, the most likely site of entry of the pathogen is generally accepted to be the oral route. Many authors have focused on the finding that, during the early phase of lymphoinvasion, the pathogen protein invades the gut mucosa through Peyer's patches and then invades the mesenteric lymph nodes, followed by the lymphoreticular system (LRS) unrelated to the digestive tract, e.g. the spleen (Maignien et al. 1999; Van Keulen et al. 2002). However, an increasing amount of data indicates that other starting points of infection are involved in prion pathogenesis, notably the respiratory system. Sheep and hamsters inoculated intranasally have been shown to develop TSE. Kincaid and Bartz (2007) have demonstrated that hamsters extranasally inoculated with a strain of transmissible mink encephalopathy agent develop the disease with an incubation period similar to that following oral inoculation. In a study dedicated to alternate ways of inoculation in sheep (Hamir et al. 2008), the authors have highlighted the presence of PrPd in lymphoid follicles of the pharyngeal tonsil
Fig. 4 Percentage of MZ innervated by $\mathrm{GFAP}+, \mathrm{NFH}+$ or NFL+ fibres. The percentage of $\mathrm{MZ}$ innervated by GFAP+, $\mathrm{NFH}+$ or NFL+ fibres was low (respectively, 5.04\%, 3.47\% and $0.56 \%$ ). Nevertheless, the statistical analyses revealed a significant increase in the number of innervated $\mathrm{MZ}$, which was correlated with ageing but without influence of the genotype

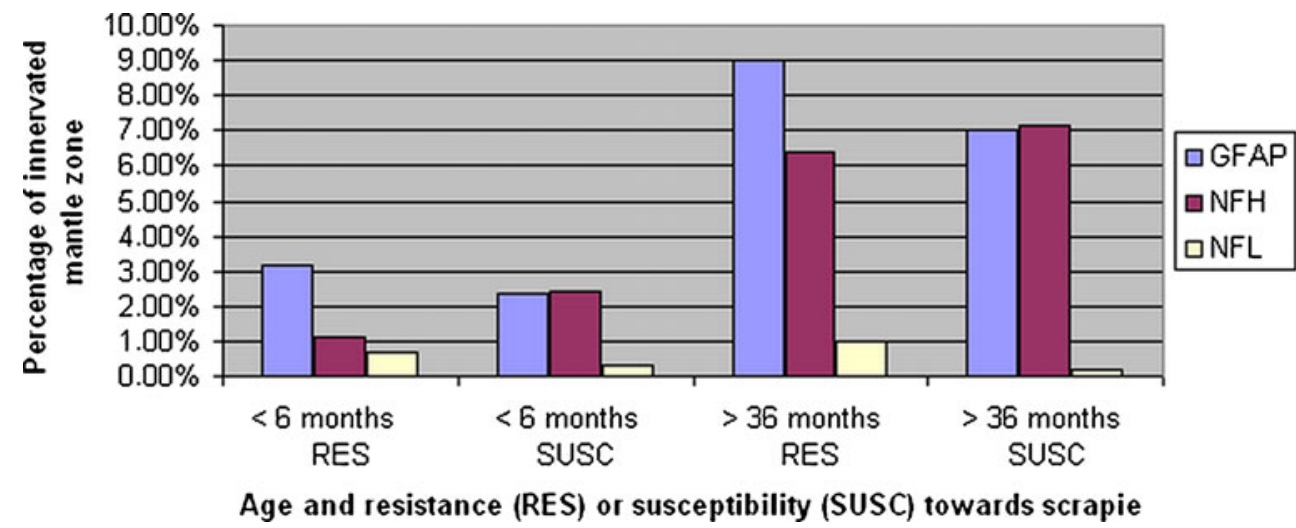



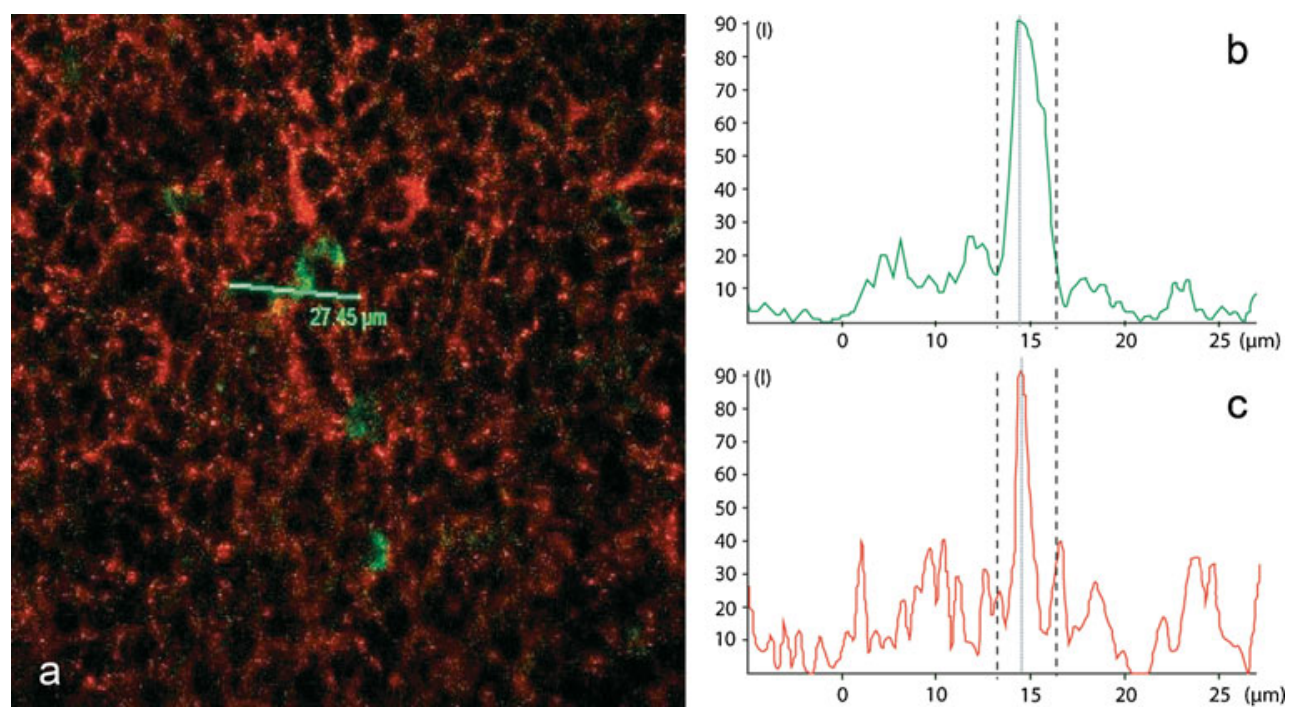

Fig. 5 Confocal analysis of co-localisation of FDCs and GFAP+ fibres within an ovine pharyngeal tonsil. Imaging (a) and spectral analysis (b, c) of close connections (yellow) between FDCs (red) and a GFAP+ fibre (green) immunostained, respectively, with FDC-B1 moAb and anti-GFAP poAb. The spectral study was carried out along the green

at 12 months after intranasal inoculation. This confirms that the pharyngeal tonsils represent a potential route for the scrapie agent dissemination.

In the lymph organs, the pathogenic agent replicates during a preclinical latent phase in the GC of the lymph follicles prior to reaching the CNS (Andreoletti et al. 2000; Heggebo et al. 2000).

Studies of mouse scrapie models have shown that mature FDCs are critical for replication in lymphoid tissues and, in their absence, neuroinvasion after peripheral challenge is significantly impaired (Brown et al. 1999; Mabbott et al. 2000). bar of $27.45 \mu \mathrm{m}$ in length traced on the selected confocal image. Superimposition of the green (b) and red (c) spectra gave a yellow signal. The spectral analyses revealed a correlation between the peaks of higher intensity in this yellow point indicating the close localisation of the FDC and the nerve fibre at this point

The intrinsic innervation of lymphoid organs and contacts between FDCs and nerve fibres might function as a bridge between the TSE replicative machinery and the peripheral nervous system (Glatzel et al. 2001). As demonstrated in the mouse experimental model, the relative positioning of FDCs and nerve endings in the spleen controls the efficiency of peripheral prion infection (Prinz et al. 2003).

This study has focused on the innervation pattern inside the various compartments of the ovine pharyngeal tonsil taking into account that (1) young lambs appear to be more susceptible than adult sheep to oral TSE infection (Jeffrey and
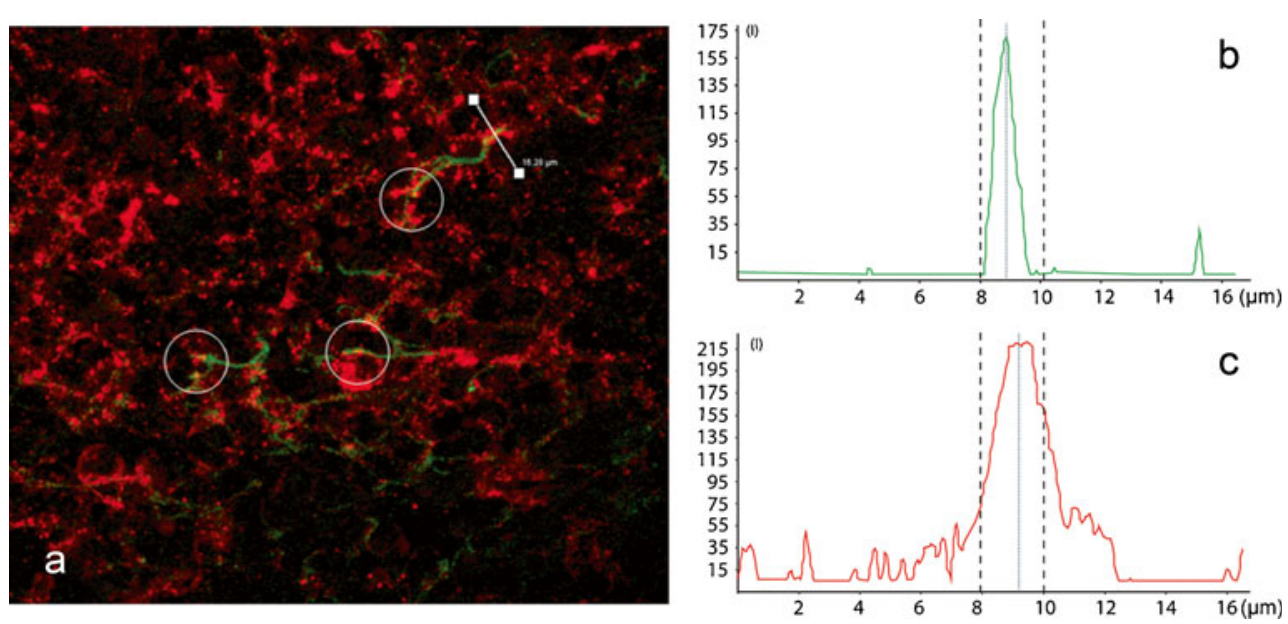

Fig. 6 Confocal analysis of co-localisation of FDCs and NFH+ fibres within an ovine pharyngeal tonsil. a Several close connections (yellow) between FDCs (red) and NFH+ fibres (green) are encircled (white circles). These structures were immunostained, respectively, with
FDC-B1 moAb and anti-NFH poAb. b, c The spectral study was carried out along the white bar of $16.28 \mu \mathrm{m}$ in length traced on the image. The correlation between the peaks of intensity at the yellow point revealed the close localisation of the labelled structures 
Gonzalez 2007) and (2) the genotype influences the susceptibility or resistance towards scrapie (Baylis et al 2002).

High innervation in high traffic area versus poor innervation in PrPd replication centres

By immunohistochemical analysis, we have shown that nerve fibres are particularly abundant in the central axis of the tonsil, towards the interfollicular area and, in older sheep, in the connective tissue underlying the respiratory epithelium. Despite poor innervation of the follicular GC, which contains the FDCs and of the MZ, which contains the membrane extensions of the FDCs, morphological analysis has indicated neuroimmune junctions between FDCs and $\mathrm{GFAP}+$ or NFH + fibres. The presence of this neuroimmune interface supports the hypothesis of the transfer of pathogen prion protein through the "FDC-nerve synapse". However, as such physical connections are rare, this mechanism of neuroinvasion is unlikely to be unique. Other lymphoid compartments in the pharyngeal tonsil might also be sites of prion neuroinvasion. The T-dependent interfollicular area, which has the top scoring of innervation, contains many immune migratory cells, notably dendritic cells able to bind and carry the pathogen protein to the FDCs or directly to nerves endings, as demonstrated in the mouse model (Dorban et al. 2007). In older sheep, the loose connective tissue underlying the respiratory epithelium is rich in GFAP+ fibres and NFH+ fibres have been detected in the lymphoepithelial tissue in proximity to $M$ cells involved in the uptake and transepithelial transport of pathogens (Casteleyn et al. 2010). Furthermore, the Schwann cells, revealed by the labelling of GFAP+ nerve fibres, are able to replicate PrPd in vitro and to transmit the disease to mice after intracerebral injection (Follet et al. 2002). They might be active in direct nerve infection. For these reasons, the interfollicular area, the connective tissue underlying the epithelium and the lymphoepithelium should be considered as key sites for the direct transfer of the pathogenic agents from the nasal cavity to the nerves endings that they contain or for neuroinvasion via mobile cells (macrophages and dendritic cells) after amplification in the GC.

These observations are corroborated by research on ovine Peyer's patches carried out by Marruchella et al. (2009) and McGovern et al. (2009). They have suggested that the rarity of FDC-nerve synapses in the lymphoid follicles of Peyer's patches throws doubt on the hypothesis that neuroinvasion is only the result of retrograde transportation of infectivity via nerves located in infected secondary follicles of the LRS.

No influence of genotype on innervation pattern

Many questions regarding the role of $\operatorname{PrPc}$ in the susceptibility to prions have been elucidated; however, the physiological role of $\operatorname{PrP}$ and the pathological mechanisms of neuroinvasion and neurodegeneration remain elusive (Flechsig and Weissmann 2004). Under physiological conditions, PrPc might notably play a role in neuronal and axonal development and in neurotogenesis through its interaction with laminin, a glycoprotein of the extracellular matrix, being involved in cell adhesion and proliferation (Cazaubon et al. 2007; Martins et al. 2001).

In sheep, the genotype at the PRNP gene and, subsequently, the intrinsic composition of the amino acids of the cellular prion protein influence the resistance/susceptibility towards scrapie. Since the nerves in the pharyngeal tonsil might be a portal of entry for prion neuroinvasion, the effect of genotype on the innervation pattern has to be evaluated.

In the present study, we have shown that the pattern of innervation is not affected by sheep genotype. Scrapie-free sheep with susceptible (ARR/VRQ or ARQ/ARQ) or resistant (ARR/ARR) genotypes exhibit the same distribution of $\mathrm{GFAP}+, \mathrm{NFH}+$ and NFL+ nerve fibres inside the various compartments of the pharyngeal tonsil. In view of our results, we can conclude that the innervation pattern is not modulated by the host $P R N P$ genotype.

\section{Differential innervation in relation to ovine age}

In agreement with those of other studies (Ciriaco et al. 1995; Madden et al. 1997), our results indicate that the innervation of the lymphoid organs depends upon the age of the sheep. Sheep older than 30 months show an increase in the number of GFAP+ and NFH+ nerve fibres in the connective tissue underlying the respiratory epithelium when compared with lambs. Similarly, Maruchella et al. (2009) have reported an increase in the number of PgP9.5+ nerve fibres in the dome area of ovine Peyer's patches underlying the intestinal epithelium in old sheep and this shows no correlation with the genotype. In both cases, and increase occurs in innervation in areas close to the site of entry of PrPd with ageing.

GC from Peyer's patches of bovines older than 24 months contain numerous NFL+ and NFH+ nerve fibres in contrast to GC of calves, which are poorly innervated (Defaweux et al. 2007).

In sheep, we have illustrated an increase with ageing of the number of GC and surrounding MZ innervated by GFAP ,$+ \mathrm{NFH}+$ and NFL + nerve fibres. In the case of scrapie, the pathogenic relevance of the innervation pattern increasing with ageing is questionable, as sheep are frequently observed to be contaminated soon after birth or at a young age (Jeffrey and Gonzalez 2007). A possible explanation for this is that, as neuroinvasion occurs in the advanced phase of infection after replication of the pathogen in the lymphoid follicles, the denser network of nerve fibres inside and around the centres of amplification of PrPd observed in older sheep might ensure more efficient infectivity. 


\section{Concluding remarks}

In conclusion, our results suggest that the transfer of prion infectivity through "FDC-nerve synapses" remains plausible, as we have demonstrated connections between both of these structures. However, the poor innervation of the lymphoid follicles supports other mechanisms of neuroinvasion, such as "dendritic cells-nerve synapses", or haematogenous or lymphatic routes.

Acknowledgements We acknowledge the technical assistance of Dr. P. Hubert. We thank Professor E. Heinen from the Human Histology Unit of The Faculty of Medicine of the University of Liege who kindly provided the FDC-B1 monoclonal antibody.

\section{References}

Andreoletti O, Berthon P, Marc D, Sarradin P, Grosclaude J, Keulen L van, Schelcher F, Elsen JM, Lantier F (2000) Early accumulation of $\operatorname{PrP}(\mathrm{Sc})$ in gut-associated lymphoid and nervous tissues of susceptible sheep from a Romanov flock with natural scrapie. J Gen Virol 81:3115-3126

Baylis M, Goldmann W, Houston F, Cairns D, Chong A, Ross A, Smith A, Hunter N, McLean AR (2002) Scrapie epidemic in a fully PrP-genotyped sheep flock. J Gen Virol 83:2907-2914

Baylis M, Chihota C, Stevenson E, Goldmann W, Smith A, Sivam K, Tongue S, Gravenor MB (2004) Risk of scrapie in British sheep of different prion protein genotype. J Gen Virol 85:2735-2740

Bessen RA, Martinka S, Kelly J, Gonzalez D (2009) Role of the lymphoreticular system in prion neuroinvasion from the oral and nasal mucosa. J Virol 83:6435-6445

Bessen RA, Shearin H, Martinka S, Boharski R, Lowe D, Wilham JM, Caughey B, Wiley JA (2010) Prion shedding from olfactory neurons into nasal secretions. PLoS Pathog 6:e1000837

Brown KL, Stewart K, Ritchie DL, Mabbott NA, Williams A, Fraser H, Morrison WI, Bruce ME (1999) Scrapie replication in lymphoid tissues depends on prion protein-expressing follicular dendritic cells. Nat Med 5:1308-1312

Casteleyn C, Van den Broeck W, Simoens P (2007) Histological characteristics and stereological volume assessment of the ovine tonsils. Vet Immunol Immunopathol 120:124-135

Casteleyn C, Cornelissen M, Simoens P, Van den Broeck W (2010) Ultramicroscopic examination of the ovine tonsillar epithelia. Anat Rec (Hoboken) 293:879-889

Cazaubon S, Viegas P, Couraud PO (2007) Functions of prion protein PrPc. Med Sci (Paris) 23:741-745

Ciriaco E, Ricci A, Bronzetti E, Mammola CL, Germana G, Vega JA (1995) Age-related changes of the noradrenergic and acetylcholinesterase reactive nerve fibres innervating the pigeon bursa of Fabricius. Ann Anat 177:237-242

Defaweux V, Dorban G, Antoine N, Piret J, Gabriel A, Jacqmot O, Falisse-Poirier N, Flandroy S, Zorzi D, Heinen E (2007) Neuroimmune connections in jejunal and ileal Peyer's patches at various bovine ages: potential sites for prion neuroinvasion. Cell Tissue Res 329:35-44

Dorban G, Defaweux V, Demonceau C, Flandroy S, Van Lerberghe PB, Falisse-Poirrier N, Piret J, Heinen E, Antoine N (2007) Interaction between dendritic cells and nerve fibres in lymphoid organs after oral scrapie exposure. Virchows Arch 451:10571065
Flechsig E, Weissmann C (2004) The role of PrP in health and disease. Curr Mol Med 4:337-353

Follet J, Lemaire-Vieille C, Blanquet-Grossard F, Podevin-Dimster V, Lehmann S, Chauvin JP, Decavel JP, Varea R, Grassi J, Fontes M, Cesbron JY (2002) PrP expression and replication by Schwann cells: implications in prion spreading. J Virol 76:2434-2439

Glatzel M, Heppner FL, Albers KM, Aguzzi A (2001) Sympathetic innervation of lymphoreticular organs is rate limiting for prion neuroinvasion. Neuron 31:25-34

Gotow T (2000) Neurofilaments in health and disease. Med Electron Microsc 33:173-199

Griffith JS (1967) Self-replication and scrapie. Nature 215:1043-1044

Halliday S, Houston F, Hunter N (2005) Expression of PrPC on cellular components of sheep blood. J Gen Virol 86:1571-1579

Hamir AN, Kunkle RA, Richt JA, Miller JM, Greenlee JJ (2008) Experimental transmission of US scrapie agent by nasal, peritoneal, and conjunctival routes to genetically susceptible sheep. Vet Pathol 45:7-11

Heggebo R, Press CM, Gunnes G, Lie KI, Tranulis MA, Ulvund M, Groschup MH, Landsverk T (2000) Distribution of prion protein in the ileal Peyer's patch of scrapie-free lambs and lambs naturally and experimentally exposed to the scrapie agent. J Gen Virol 81:2327-2337

Horiuchi M, Yamazaki N, Ikeda T, Ishiguro N, Shinagawa M (1995) A cellular form of prion protein (PrPC) exists in many non-neuronal tissues of sheep. J Gen Virol 76:2583-2587

Hunter N, Goldmann W, Foster JD, Cairns D, Smith G (1997) Natural scrapie and PrP genotype: case-control studies in British sheep. Vet Rec 141:137-140

Hunter N, Foster J, Chong A, McCutcheon S, Parnham D, Eaton S, MacKenzie C, Houston F (2002) Transmission of prion diseases by blood transfusion. J Gen Virol 83:2897-2905

Jeffrey M, Gonzalez L (2007) Scrapie. In: Aitken ID (ed) Diseases of sheep, 4th edn. Blackwell, Edinburgh, pp 242-250

Jeffrey M, McGovern G, Goodsir CM, Brown KL, Bruce ME (2000) Sites of prion protein accumulation in scrapie-infected mouse spleen revealed by immuno-electron microscopy. J Pathol 191:323-332

Jessen KR, Morgan L, Stewart HJ, Mirsky R (1990) Three markers of adult non-myelin-forming Schwann cells, 217c(Ran-1), A5E3 and GFAP: development and regulation by neuron-Schwann cell interactions. Development 109:91-103

Kincaid AE, Bartz JC (2007) The nasal cavity is a route for prion infection in hamsters. J Virol 81:4482-4491

Kraehenbuhl JP, Neutra MR (2000) Epithelial M cells: differentiation and function. Annu Rev Cell Dev Biol 16:301-332

Lee MK, Cleveland DW (1996) Neuronal intermediate filaments. Annu Rev Neurosci 19:187-217

Mabbott NA, Mackay F, Minns F, Bruce ME (2000) Temporary inactivation of follicular dendritic cells delays neuroinvasion of scrapie. Nat Med 6:719-720

Madden KS, Bellinger DL, Felten SY, Snyder E, Maida ME, Felten DL (1997) Alterations in sympathetic innervation of thymus and spleen in aged mice. Mech Ageing Dev 94:165-175

Maignien T, Lasmezas CI, Beringue V, Dormont D, Deslys JP (1999) Pathogenesis of the oral route of infection of mice with scrapie and bovine spongiform encephalopathy agents. J Gen Virol 80:3035-3042

Marruchella G, Ligios C, Baffoni M, Cancedda MG, Demontis F, Donatucci G, Chiocchetti R, Clavenzani P, Lalatta-Costerbosa G, Di Guardo G (2009) Ileal tract and Peyer's patch innervation in scrapie-free versus scrapie-affected ovines. Arch Virol 154:709-714

Martins VR, Mercadante AF, Cabral AL, Freitas AR, Castro RM (2001) Insights into the physiological function of cellular prion protein. Braz J Med Biol Res 34:585-595 
McGovern G, Martin S, Gonzalez L, Witz J, Jeffrey M (2009) Frequency and distribution of nerves in scrapie-affected and unaffected Peyer's patches and lymph nodes. Vet Pathol 46:233-240

Melot F, Defaweux V, Jolois O, Collard A, Robert B, Heinen E, Antoine N (2004) FDC-B1: a new monoclonal antibody directed against bovine follicular dendritic cells. Vet Immunol Immunopathol 97:1-9

Miller MW, Williams ES, Hobbs NT, Wolfe LL (2004) Environmental sources of prion transmission in mule deer. Emerg Infect Dis 10:1003-1006

Notturno F, Caporale CM, De Lauretis A, Uncini A (2008) Glial fibrillary acidic protein: a marker of axonal Guillain-Barre syndrome and outcome. Muscle Nerve 38:899-903

Prinz M, Heikenwalder M, Junt T, Schwarz P, Glatzel M, Heppner FL, Fu YX, Lipp M, Aguzzi A (2003) Positioning of follicular dendritic cells within the spleen controls prion neuroinvasion. Nature 425:957-962

Prusiner SB (1982) Novel proteinaceous infectious particles cause scrapie. Science 216:136-144
Race R, Jenny A, Sutton D (1998) Scrapie infectivity and proteinase K-resistant prion protein in sheep placenta, brain, spleen, and lymph node: implications for transmission and antemortem diagnosis. J Infect Dis 178:949-953

Sbriccoli M, Cardone F, Valanzano A, Lu M, Graziano S, De Pascalis A, Ingrosso L, Zanusso G, Monaco S, Bentivoglio M, Pocchiari M (2009) Neuroinvasion of the $263 \mathrm{~K}$ scrapie strain after intranasal administration occurs through olfactory-unrelated pathways. Acta Neuropathol 117:175-184

Seeger H, Heikenwalder M, Zeller N, Kranich J, Schwarz P, Gaspert A, Seifert B, Miele G, Aguzzi A (2005) Coincident scrapie infection and nephritis lead to urinary prion excretion. Science 310:324-326

Van Keulen LJM, Vromans MEW, Van Zijderveld FG (2002) Early and late pathogenesis of natural scrapie infection in sheep. APMIS 110:23-32

Vascellari M, Nonno R, Mutinelli F, Bigolaro M, Di Bari MA, Melchiotti E, Marcon S, D'Agostino C, Vaccari G, Conte M, De Grossi L, Rosone F, Giordani F, Agrimi U (2007) PrPSc in salivary glands of scrapie-affected sheep. J Virol 81:4872-4876

Weissmann C (2004) The state of the prion. Nat Rev Microbiol 2:861-871 